\title{
Article \\ Materials Inspiring Methodology: Reflecting on the Potential of Transdisciplinary Approaches to the Study of Archaeological Glass
}

\author{
Sara Fiorentino*, Tania Chinni and Mariangela Vandini \\ Department of Cultural Heritage, Ravenna Campus, University of Bologna, 40126 Bologna, Italy; \\ tania.chinni2@unibo.it (T.C.); mariangela.vandini@unibo.it (M.V.) \\ * Correspondence: sara.fiorentino2@unibo.it
}

Citation: Fiorentino, S.; Chinni, T.;

Vandini, M. Materials Inspiring

Methodology: Reflecting on the

Potential of Transdisciplinary

Approaches to the Study of

Archaeological Glass. Appl. Sci. 2021,

11, 8049. https://doi.org/10.3390/

app11178049

Academic Editor: Asterios Bakolas

Received: 21 July 2021

Accepted: 27 August 2021

Published: 30 August 2021

Publisher's Note: MDPI stays neutral with regard to jurisdictional claims in published maps and institutional affiliations.

Copyright: (c) 2021 by the authors. Licensee MDPI, Basel, Switzerland. This article is an open access article distributed under the terms and conditions of the Creative Commons Attribution (CC BY) license (https:/ / creativecommons.org/licenses/by/ $4.0 /)$.

\begin{abstract}
The contribution that materials science has made to the understanding of ancient glassmaking is unquestionable, as research undertaken in recent decades has extensively demonstrated. Archaeological glass is far from being a homogeneous class of materials, encompassing objects made for different uses, manufactured in different periods and geographic areas, with a variety of tools and working techniques. If all these factors are not adequately considered when approaching the study of ancient glasses from an archaeometric perspective, data obtained by analyses can incur the risk of being less informative or even misinterpreted. Moving from previously performed research, this paper is aimed at reflecting on the potential of synergistic approaches for the study of archeological glasses, based on the interrelation among different disciplines and fostering the integration of archaeological and historical knowledge with data-driven scientific analyses.
\end{abstract}

Keywords: archaeological glass; archaeometry; VIS-RS; SEM-EDS; Raman spectroscopy; XRPD; EPMA; LA-ICP-MS

\section{Introduction}

The study of objects belonging to our material culture cannot ignore a persevering and constructive dialogue between different fields of knowledge. Only a well-integrated, transdisciplinary approach can pave the way for an in-depth understanding of historical, cultural, socioeconomic, and technological issues for which the objects of our past are silent witnesses.

Although in the last few decades there has been an increasing tendency towards combined archaeological and archaeometric studies [1,2], this has not always resulted in long-lasting cooperation among disciplines. On the one hand, archaeology often turns to archeometry to answer open questions mainly related to the provenance and dating of the objects or as a scientific support to confirm previously formulated hypotheses. On the other hand, archaeometric studies leave a space that is not always adequate for archaeological and/or historiographic considerations, sometimes leading to a discussion of extreme detail of a single analytical data which, however, is detached from a broader contextualisation. The above considerations are also valid for archaeological glass, a category whose applied research is still affected by a subdivision of roles based on the specific knowledge in a particular field as well as by a lack of systematic integration of data across disciplines. All this to the detriment of a real joint research action, which is fundamental for understanding objects from the past beyond their material components and as vectors of culture. More specifically, though a noticeable broadening of the glass research community has occurred in the last decades, the need to move from a traditional data gathering to a more interpretative phase has been highlighted [3]. Research should guide toward a better understanding of the reasons and practices behind the compositional and technical developments of the ancient glass industry, in the attempt to deepen the current understanding of glass production, 
trade, and technologies and to contextualise this specific material within the sociocultural and economic framework of past societies. The discernment of such a multifaceted scenario cannot ignore a synergistic and complementary approach between different disciplines. A sound integration of analytical data into archaeological and, by extension, anthropological research, is the keystone that can lead to a more comprehensive understanding of ancient glass industries and related technological aspects.

The establishment of an enduring transdisciplinary approach could overcome several critical issues affecting applied research on material culture, with specific reference to glass production. In the first place, one should be cautious in comparing archaeometric data relating to different categories of objects (i.e., tableware with mosaic tesserae and/or ornaments), as sociocultural and economic reasons behind the manufacture of these objects could lead to erroneous conclusions. Comparing objects destined for different uses, albeit coming from the same context, can mislead the interpretation of archaeometric data and lead to hypotheses that are not sustainable from an archaeological perspective. Hence, there is a need to overcome the traditional method of comparison, mainly based on associating data pertinent to types of glass objects very different from each other. Another critical issue to pay particular attention to is the number of finds to be analysed: obtaining conspicuous datasets should not be the key criterion when selecting the finds. If the analysed fragments are not "diagnostic", meaning they are not attributable to distinctive typologies with verified chronology, the achieved dataset, though numerically consistent, might not provide useful information. Prior to the analytical phase, it is of primary importance to understand the context where the finds were unearthed, as well as the related sociocultural aspects.

As a consequence, an actual co-participatory approach between disciplines related to different research fields is the only tool capable of overcoming the limits that, by its intrinsic nature, each discipline considered individually has. Without prejudice to the need for everyone to work in their own specific field of study, it is necessary to overcome the still feebly implemented reading and synergistic discussion of the results that emerge from the various disciplinary areas involved.

Through an examination of selected previously studied assemblages from different types of contexts, this paper aims to reflect on the potentialities stemming from a transdisciplinary approach applied to the study of archaeological glass. Whatever the research field, ancient glasses are strongly heterogeneous materials: from a historical-archaeological perspective, glass assemblages from excavated contexts comprise different objects intended for different uses (i.e., glassware, ornaments, and architectural decorations), often made in different places and workshops, using different tools and techniques. Should these elements not be properly taken into consideration, data obtained by archaeometric analyses could incur the risk of being uninformative. Framed in a broader context and without any intent to criticise previous research, this paper fosters the promotion of a synergistic approach in the study of archaeological glass, setting the basis for an enduring integration of different disciplines.

\section{Materials and Methods}

Previously studied assemblages of archaeological glass available at the Conservation Science Laboratory of the Department of Cultural Heritage (University of Bologna-Ravenna Campus) have been selected for this study: 13th-14th century ampoules, nuppenbecher and kropfflasche from the monastic complex of San Severo (Classe, Ravenna, Italy) [4]; 14th-16th century gambassini drinking vessels from the monastic complex of San Severo (Classe, Ravenna, Italy) and from the Rontana Castel (Brisighella, Ravenna, Italy) [5]; 8th century mosaic glass tesserae from the qasr of Khirbet al-Mafjar (Jericho, Palestine) [6]; and 8th century mosaic glass tesserae from the Great Mosque of Damascus (Syria) $[7,8]$. When other assemblages of materials from previous studies which have not been carried out by the authors are taken as examples, references are provided in the text.

An Olympus S761 stereomicroscope (magnification up to 45×, Olympus Corporation, Shinjuku, Tokyo, Japan) associated with an Olympus Soft Imaging Solutions GMBH model 
SC100 camera (Olympus Corporation, Shinjuku, Tokyo, Japan) was used for preliminary morphological observations and documentation.

For Visible Reflectance Spectrometry (VIS-RS), a MINOLTA CM-2600d portable spectrometer was used. The system is equipped with an internal integrating sphere of $56 \mathrm{~mm}$ diameter, in reflectance geometry d: $8^{\circ}$, with three Xenon pulsed lamps, and a D65 illuminant was used; calibration was performed against a $\mathrm{BaSO}_{4}$ standard plate; the spectral range is $400-700 \mathrm{~nm}$, with a spectral resolution of $10 \mathrm{~nm}$ and the area of sight of $3 \mathrm{~mm}$ diameter. Spectra Magic software was employed to elaborate data; specular component excluded (SCE/0) was selected, according to the literature [9].

Microsamples were taken from either objects or diagnostic fragments for performing micromorphological, microtextural, and compositional analyses. Sections were prepared by embedding microsamples in a polyester resin then polished by using abrasive papers of decreasing grain in order to expose the embedded samples avoiding any losses.

A Scanning Electron Microscope (SEM) back-scattered electron signal (BSE) was used for the inspection of the morphological features of the inclusions coupled with Energy Dispersive Spectroscopy (EDS) spot measurements to achieve a preliminary qualitative and semiquantitative elemental analysis of the inclusions themselves. Analyses were carried out on polished and carbon-coated sections. Images and EDS spectra were collected on carboncoated crosssections, using an ESEM FEI Quanta 200, equipped with an EDAX energy dispersive spectrometer. Analyses were performed in high-vacuum using an acceleration voltage of $25 \mathrm{kV}$ and an energy resolution of $\sim 200 \mathrm{eV}$; working distance was set at $10 \mathrm{~mm}$, spot size was between four and five $\mu \mathrm{m}$.

Micro-Raman spectra were collected by using a Bruker Senterra dispersive Raman spectrometer equipped with an integrated Olympus BX40 microscope. A $785 \mathrm{~nm} \mathrm{He}-$ Ne laser was employed, in the $300-3500 \mathrm{~cm}^{-1}$ region. Analytical measurements were performed with a $50 \times$ long working distance objective, operating at a power of $10 \mathrm{~mW}$ (red and blue samples) or $25 \mathrm{~mW}$ (yellow and green samples) with a spectral resolution of $3.5 \mathrm{~cm}^{-1}$. Raman measurements were performed on polished sections after carboncoating removal.

X-ray Powdered Diffractometry (XRPD) analyses were performed on finely powdered samples manually pressed on a Ag sample holder in a Rigaku Miniflex diffractometer employing $\mathrm{CuK} \alpha_{1}$ radiation in the range $2 \theta: 4^{\circ}-64^{\circ}, \theta$ scan speed: $1^{\circ} \mathrm{min}^{-1}$.

To determine the bulk chemistry of all samples under study, Electron Probe Microanalysis (EPMA) was performed on polished and carbon-coated sections. Chemical analyses of major and minor elements $(\mathrm{Na}, \mathrm{Mg}, \mathrm{Al}, \mathrm{Si}, \mathrm{P}, \mathrm{S}, \mathrm{Cl}, \mathrm{K}, \mathrm{Ca}, \mathrm{Ti}, \mathrm{Cr}, \mathrm{Mn}, \mathrm{Fe}, \mathrm{Co}, \mathrm{Cu}, \mathrm{Sn}$, $\mathrm{Sb}$, and $\mathrm{Pb}$ ) were performed using a CAMECA-CAMEBAX equipped with four scanning Wavelength Dispersive Spectrometers (WDS). A beam current of $2 \mathrm{nA}$, an acceleration voltage of $20 \mathrm{kV}$, and a spot size of $5 \mu \mathrm{m}$ were used for $\mathrm{Na}, \mathrm{K}, \mathrm{Si}$, and $\mathrm{Al}$; for all other elements, a beam current of $20 \mathrm{nA}$, an acceleration voltage of $20 \mathrm{kV}$, and a spot size of $1 \mu \mathrm{m}$ were used. Synthetic pure oxides were used as standards for $\mathrm{Al}, \mathrm{Cr}, \mathrm{Fe}$, and $\mathrm{Sn}$, synthetic $\mathrm{MnTiO}_{3}$ for $\mathrm{Mn}$ and $\mathrm{Ti}$, wollastonite for $\mathrm{Si}$ and $\mathrm{Ca}$, albite for $\mathrm{Na}$, periclase for $\mathrm{Mg}$, $\mathrm{PbS}$ for $\mathrm{Pb}$, orthoclase for $\mathrm{K}$, apatite for $\mathrm{P}$, sphalerite for $\mathrm{S}, \mathrm{Sb}_{2} \mathrm{~S}$ for $\mathrm{Sb}$, and pure elements for $\mathrm{Co}, \mathrm{Cu}$, and Ni. SMITHSONIAN GLASS A standard [10] was also employed as a reference sample. Ten points were analysed on each sample, and the mean values were calculated. The measured accuracy for the analysed elements was better than $3 \%$. The standard deviations among the analysed points resulted in between $1 \%$ and $3 \%$ and $3 \%$ and $5 \%$ for major and minor constituents, respectively. The detection limit for the minor elements was between $0.01 \mathrm{wt} \%$ and $0.04 \mathrm{wt} \%$. The correction program was based on the PAP method [11] and was used to process the results for matrix effects.

Laser Ablation-Inductively Plasma Coupled-Mass Spectrometry (LA-ICP-MS) was carried out to determine the concentration of 37 trace elements. Analyses were performed by a Thermo Fisher X-Series II quadrupole based ICP-MS coupled with a New Wave ablation system with a frequency quintupled $(\lambda=213 \mathrm{~nm}) \mathrm{Nd}$ :YAG laser. Laser repetition rate and laser energy density on the sample surface were fixed at $20 \mathrm{~Hz}$ and $\sim 18 \mathrm{~J} / \mathrm{cm}^{2}$, 
respectively. Analyses were carried out using a laser spot diameter of $100 \mu \mathrm{m}$ on the same polished samples used for EPMA after carbon-coating removal. Due to the highly heterogeneous microstructure of the tesserae, six points were analysed on each sample and the mean values were then calculated. External calibration was performed using NIST 610 and 614 glass as external standards; NIST 612 was also used as a secondary reference sample to check precision and accuracy (Pearce et al. 1997). ${ }^{29} \mathrm{Si}$ was employed as an internal standard, whose concentration was determined by EPMA following the method proposed by Longerich and colleagues [12]. The distribution of REE and of the other trace elements was analysed by normalising the data to the upper continental crust [13].

\section{Results and Discussion}

\subsection{Understanding Archaeological Context: The First Step toward a Tailored Selection of Materials}

The most recurring questions related to the study of archaeological materials are aimed at understanding whether a local production can be identified. This issue is of a particular relevance for glass, where the very sporadic occurrence of furnace remains makes it extremely challenging to map the production sites and distinguish them from the working sites. If the context where materials have been unearthed shows evidence of a production/working activity of glass (i.e., furnace remains, production waste, and tools), the selection of the assemblage to be investigated is generally aimed to characterise all these indicators. Therefore, the choice to carry out archaeometric analyses on materials that can be typologically heterogeneous (such as production indicators, scraps, and finished objects) is justified by the need to identify raw materials used in glassmaking. An example is, in this regard, the ancient port of Classe (Ravenna, Italy), one of the most important trade centres between the 5th and 8th centuries CE in the Northern Adriatic area. Archaeological excavations identified the main context for glassworking inside one of the warehouses built at the beginning of the 5th century CE, where a small circular kiln had been unearthed; around the kiln, a massive amount of glass fragments and glassworking waste (973 finds) was brought to light in 2001 [14]. Results from archaeometric analyses demonstrated that, in the 5th century CE, the secondary glass workshop in Classe was dedicated to the shaping of vessels starting from raw glass chunks and, possibly, cullets. Evidence of glass remelting and glassblowing were found, while no evidence of primary production was uncovered [15]. Moreover, comparisons between Classe and Aquileia, the two most important Late Antique archaeological sites of Northeastern Italy, were undertaken to shed light on the role of the Northern Adriatic area in glass production, trade, and consumption. The comparison showed that, unlike Classe, correlations did not exist in Aquileia between specific types of objects and the compositions of glass; this data has further strengthened the hypothesis of a local shaping of specific objects at Classe [16].

An analogous approach in the selection of materials to be studied can be applied to cargos from shipwrecks. The case of the wreck of the Iulia Felix ship, which sank in the 2nd century CE off the coast of Grado (Friuli-Venezia Giulia, Italy) is, in this sense, thorough. The archaeological and archaeometric study of the assemblage of glass materials was performed on cullets and finished objects to achieve data on colourless and coloured Roman glass production technology. The strong evidence of compositional variability among the Iulia Felix assemblage strengthened the hypothesis of a dispersed production model for Roman glassware and the common practice of recycling in Roman ages, especially for daily-use vessels $[17,18]$.

If archaeological glass is found at multilayered contexts and/or where there is no univocal evidence of local production, the approach to the selection of materials to be studied needs to be different. Multilayered contexts are particularly challenging to be approached: inhabited for more or less extended periods, they generally are urban, monastic, and/or residential sites, where structures that can be interpreted as small kilns, though without sound evidence of glass production (i.e., no production/processing indicators recovered), can also be found. When these contexts are faced, an archaeometric study of numerically consistent sets of fragments (without paying due attention to the multilayered structure of 
the site and often comparing data obtained from the analysis of fragments pertaining to different objects and chronological frames) can rarely result in a meaningful and exhaustive scenario. The result is often a multiplicity of hypotheses regarding the import of objects from other sites and, when no comparisons can be found with other sites, the risk is to hypothesise local productions without sound archaeological evidence.

Recent studies have highlighted the potential associated with adopting a synergistic transdisciplinary approach when working on multilayered contexts. The starting point is represented by an in-depth tailored archaeological study of the assemblage functional to identify distinctive shapes and their chronology. The results obtained by chronotypological study of the objects can, in fact, outline different scenarios to assess; the most common are those characterised by a) specific groups of objects with the same chronology; (b) a greater statistical incidence of a specific distinctive shape (or a few of them). Depending on whether the chronotypological study of the finds identifies one or the other scenario, key issues to which the archaeometric analyses will be called to contribute will be evaluated. An exhaustive example of the approach centred on the study of specific types of objects falling within the same chronological frame is provided by Sedlackova and colleagues [19]. The study aimed to investigate the production and import of glass objects in Bratislava involving finds of tableware and window glass. The preliminary archaeological study allowed the attribution of the finds to three main timeframes verified by the occurrence of distinctive shapes and decorative features: 13th, late 13th-14th and mid-15th century CE. The subsequent archaeometric investigations made it possible, for each of the examined periods, to ascertain the composition of the glass and to establish comparisons with chronologically compatible Central Europe productions shedding light on commercial and political orientations. The study is an example of how informative data can also be obtained by working on a small number of fragments, as long as well contextualised from a chronotypological perspective to set up comparison with the same types of objects found in other geographical areas.

The same methodological approach has been applied to a first selection of materials from the monastic complex of San Severo in Classe (Ravenna, Italy) [4]. The chronotypological study of the material identified the occurrence of three particular types of glass objects, although not present in statistically high numbers: ampoules, nuppenbecher, and kropfflasche, dated to the 13th-16th century CE. The close similarities, in terms of chemical features, between these objects from San Severo and comparable finds from Czech Republic, further strengthened the hypothesis of commercial contact between Central Europe and Italy in the Middle Ages. The scope of both studies, focused on a few distinctive shapes, was to trace, through an interrelation between chronotypological and archaeometric study, contacts between two geographically distant areas, united by the occurrence of the same types of glass objects. The monastic complex of San Severo can be taken as an example of another approach applicable to archaeological glass, based on the study of one (or a few) statistically relevant shape(s). This is the case of the daily-use drinking glass beaker known as gambassino [5]. Recently published research aimed at deepening the historical, archaeological, and compositional knowledge between the variants of this object, found in two contemporary sites located at a distance of about $50 \mathrm{~km}$ from each other: the monastery of San Severo and the Rontana castle (Brisighella, Ravenna, Italy). For both sites, the gambassino was the shape with the greatest statistical impact, accounting for over $70 \%$ of the entire assemblage of unearthed glass materials [14]. Moving from a local scale, the study then expanded to regional and transregional comparisons involving central-northern Italy and the Balkan side of the Adriatic Sea. Thanks to an interrelation among archaeological, historical, and archaeometric data, evidence has been underpinned for the movement of finished objects, raw materials, and artisans in the considered timeframe: the displacement of artisans and the circulation of raw materials, the recurrence of this beaker in numerous excavation contexts in the northern Adriatic area, as well as the identification of compositional groups different from each other but overlapping among assemblages, has led to the 
hypothesis of a "widespread production" model of the common drinking beaker known as gambassino.

The aforementioned studies stand as examples of the great variety of approaches applicable to the study of archaeological glass. The selection of one or the other approach cannot be separated from the in-depth knowledge of the archaeological context, paying particular attention to the type of settlement (i.e., urban, rural, residential, or monastic) and to its continuity of life across time. Being aware of the context is, thus, the first step to be taken. The next step is the in-depth study of the finds, of the way they are linked to the chronology of the site, and of their possible connections with other contexts. All these elements contribute to the definition of the questions underlying the selection of the samples to undergo archaeometric analyses, so that they can provide suitable data to support hypotheses of production and/or provenance of the artefacts under study.

\subsection{Archaeometric Analyses: Defining the Analytical Approach Based on Material Features}

Once the selection of materials to be analysed has been completed, the setup of the analytical protocol may vary according to the features of the materials themselves. An indepth discussion of the potential and limits of all possible analytical techniques applicable to the study of archaeological glass would be beyond the scope of this article. Therefore, in the following discussion, reference will be made to the most commonly used analytical techniques for archaeometric studies on glass to foster data comparability.

The first criterion influencing the choice of analytical protocol is whether the materials to be analysed are naturally or intentionally coloured glasses. The archaeometric approach to the study of nonintentionally coloured glass (which means without the intentional addition of colouring and opacifying agents to the base glass) is simpler than deeply coloured (and often opaque) glass. The latter is, in fact, characterized by greater heterogeneity in terms of microstructure and compositional features, due to the addition of specific raw materials aimed at imparting the desired colour and degree of opacity. The setup of a more articulated analytical approach is needed based on the interrelation of different investigation techniques to achieve an in-depth characterization of colouring and opacifying agents. When dealing with naturally coloured glass, the archaeometric approach is based on the use of fewer analytical techniques, the primary objective being the determination of the chemical composition of the base glass and, if needed, the provenance of the materials used as vitrifying agents. Figures 1 and 2 show block diagrams of the two different analytical approaches applicable to the study of naturally and intentionally coloured glass; it is always advisable to carry out an observation and documentation of the samples in optical microscopy, preliminary to the filing of the fragments/objects under study.

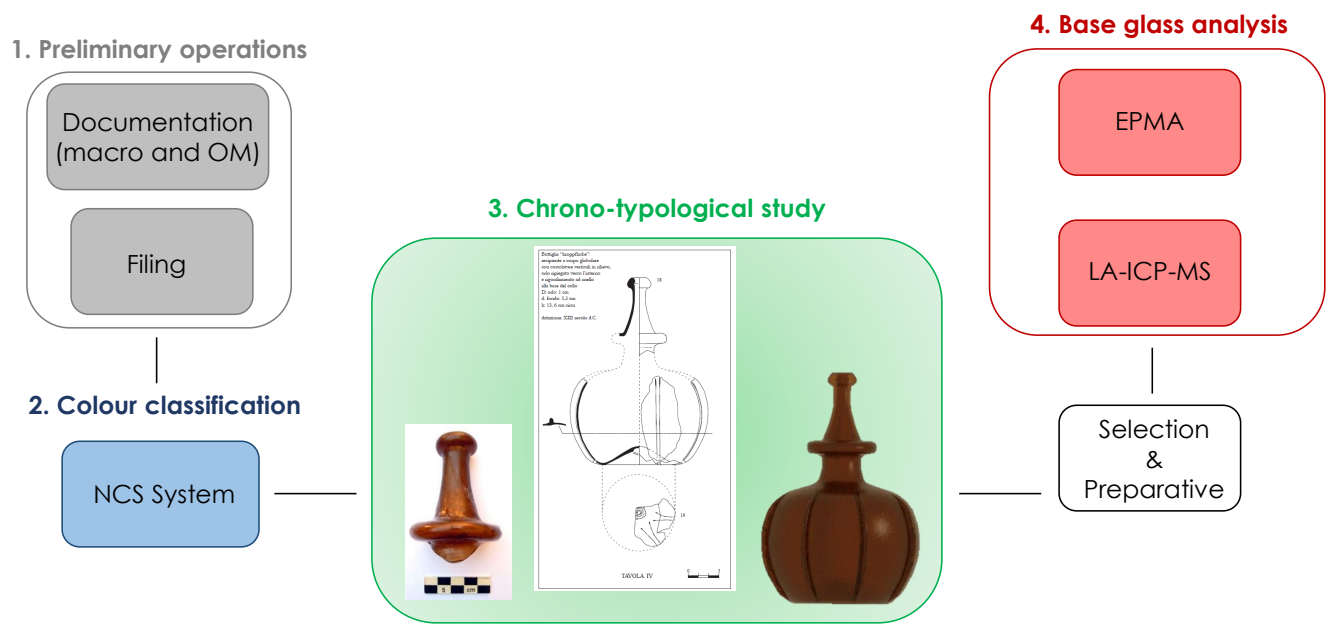

Figure 1. Block diagram of the proposed analytical approach for naturally coloured archaeological glass. 


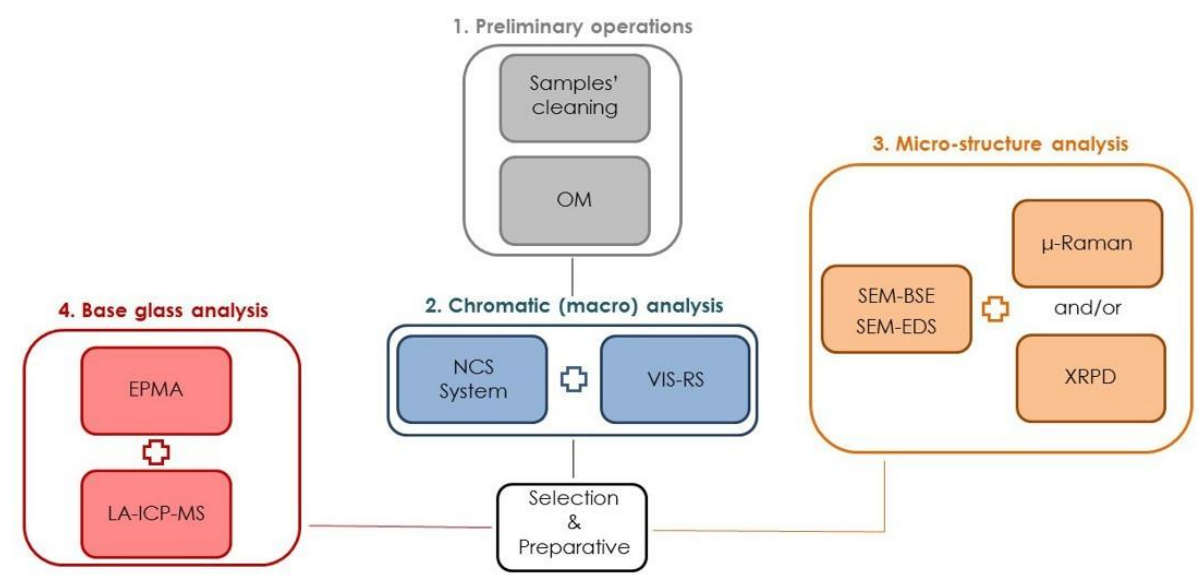

Figure 2. Block diagram of the proposed analytical approach for coloured archaeological glass.

When archaeometric analyses have to be performed on intentionally coloured glasses, it is highly recommended to start with an in-depth study of the colouring (and opacifying) phases. As more extensively discussed elsewhere [8], since deeply coloured glasses show highly heterogeneous microstructures and microtextures, a thorough characterisation of colouring and opacifying phases should be carried out before the bulk composition of the vitreous matrix to avoid misunderstandings in data processing and interpretation.

A combined Standard Colour System Chart (such as NCS Index or PANTONE) and Visible Reflectance Spectroscopy (VIS-RS) approach is here proposed as a starting point. It can support, in fact, an objective definition of the chromatic hues and shades of deeply coloured glasses avoiding any subjective nomenclature. The example par excellence is represented, in this case, by a particular category of glass-based artefacts: mosaic tesserae. For these small cubes of coloured glass, a chronotypological study is unfeasible; therefore, colours and opacity are the only macrofeatures that can be used to methodically select among copious assemblages of samples to be studied. NCS coordinates can be used to effectively separate the tesserae into preliminary chromatic macrocategories (i.e., yellow, green, blue, red, and black). These groups can be defined based on NCS coordinates and, more precisely, by taking the second part of the NCS-notation into account, which describes the hue by means of a numerical code. For example, a tessera with NCS-notation S 2030-G70Y will be described as of a yellow colour and code G70Y indicating a colour shade described as a yellow $(\mathrm{Y})$ with $70 \%$ resemblance to yellow and a 30\% resemblance to green $(\mathrm{G})$. It is possible, in this way, to avoid the use of definitions such as "greenish yellow" or "yellowish green", eliminating a first important degree of subjectivity in the definition of colour. For NCS evaluations to be accurate, it is important that observations are made in controlled repeatable daylight conditions avoiding any artificial source of light.

After preliminary NCS-aided discrimination between chromatic macrocategories, further data on optical properties $\left(\mathrm{L}^{*} \mathrm{a}^{*} \mathrm{~b}^{*}\right.$ numerical coordinates and the reflectance for each wavelength in the visible spectrum) can be collected by VIS-RS. Figure 3 shows a comparison between reflectance curves acquired on tesserae belonging to Red, Yellow, Green, and Blue chromatic macrocategories identified by taking NCS-notations into account. Reflectance curves of tesserae belonging to the NCS-Red macrocategory displayed a very flat behaviour in the wavelength range between 400 and $580 \mathrm{~nm}$, followed by an increase in reflectance intensity for the wavelengths above $580 \mathrm{~nm}$. NCS-Blue and NCS-Green tesserae showed bell-shaped reflectance curves; the reflectance peak was located in the region between 440 and $540 \mathrm{~nm}$ for the blue tesserae, while, for the green tesserae, it was slightly shifted between 470 and $540 \mathrm{~nm}$. Last, for the NCS-Yellow tesserae, reflectance curves were characterised by an increase in reflectance intensity for the wavelengths above $560 \mathrm{~nm}$. Though further research is needed, the potentiality of VIS-RS seems to go far beyond the description of colours by means of reflectance curves and $\mathrm{L}^{*} \mathrm{a}^{*} \mathrm{~b}^{*}$ numerical coordinates. In particular, the shapes of the reflectance curves in the visible spectrum and 
the percentages of reflectance can deliver preliminary qualitative information relating to the colouring and opacifying agents $[6,20,21]$.

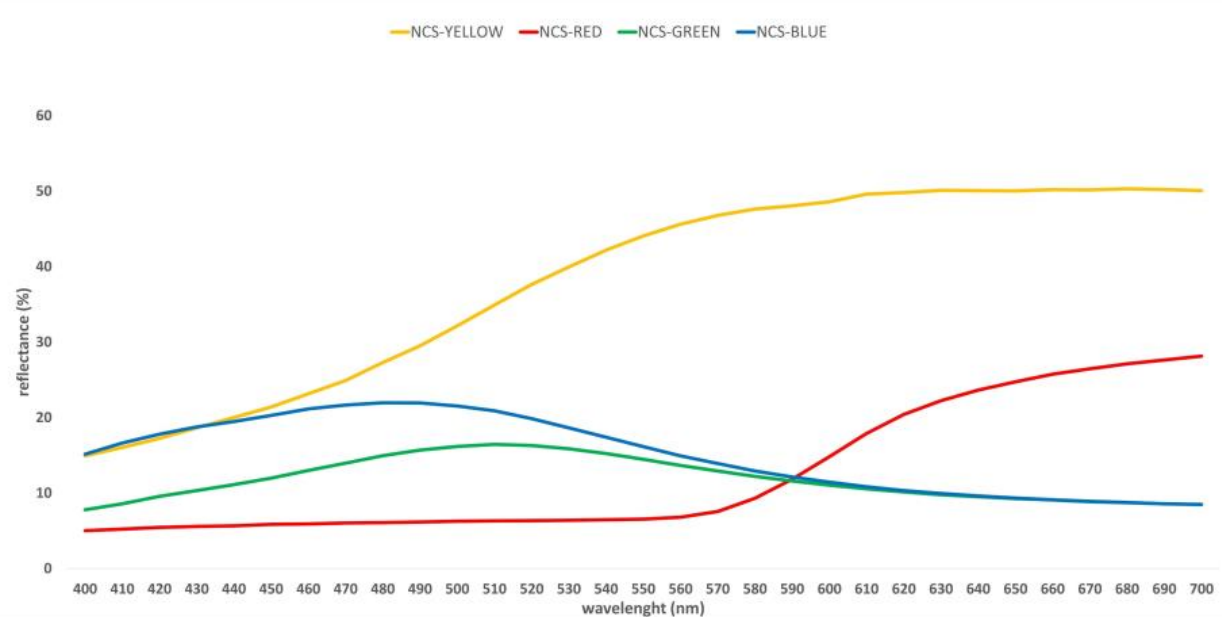

Figure 3. Comparison between reflectance curves acquired by VIS-RS on NCS-Red, Yellow, Green, and Blue tesserae.

After preliminary OM documentation, sampling, embedding, and polishing, SEMEDS should be performed on deeply coloured glass. Back-scattered electrons (BSE) signals allow detecting and documenting of the different morphologies of the crystals precipitated into the glassy matrix, with EDS spot measurements also ascertaining their elemental composition. SEM-EDS is undoubtedly suitable to carry out high magnification morphological inspection of the inclusions dispersed in glassy matrix, as well as providing a qualitative and semiquantitative analysis of their elemental composition. However, to provide a more in-depth characterisation of these inclusions, necessary to identify raw materials responsible for the colour and opacity of the tesserae, SEM-EDS inspection needs to be integrated with other analytical techniques.

Especially if coloured and opaque, archaeological glasses are strongly heterogeneous materials; it is, thus, quite challenging to define what is the most suitable analytical technique for providing a full characterisation of the inclusions. It would, maybe, be more correct to claim that there is not only one. The choice of the technique/s is highly dependent upon the nature of the inclusions that we want to investigate. An integration of at least one molecular (such as Raman Microscopy) and one mineralogical analysis (X-ray Powder Diffraction-XRPD, micro-XRD or SEM equipped with an Electron Backscattered Diffraction detector-EBSD, if available) is recommended, offering an appropriate compromise to achieve a thorough characterisation of the inclusions responsible for the colour and opacity of glass.

The characterisation of lead-tin-antimony-based compounds found in ancient glasses as colouring and opacifying agent can be taken as an example. As discussed elsewhere in more detail [8], lead-tin-antimonate crystals have been attested to in several assemblages of opaque coloured glasses [22-25]. The most credited hypothesis is that the presence of lead-antimonate inclusions doped with tin could be related to the use of tin-rich metallurgical scraps [26,27]; therefore, an exact characterisation of these inclusions could represent the starting point for providing insights into the identification of a possible area of origin of the raw materials used as colouring and opacifying phases. SEM-BSE images (Figure 4a) provide information on the morphology of the inclusions, showing the occurrence of micrometric anhedral crystals in the vitreous matrix, frequently clustered together; EDS spot measurements carried out on the crystals gave preliminary information on the elemental composition of the crystals, demonstrating that they were mainly made of antimony and lead, although tin could also be detected (Figure 4b). To achieve an exact characterisation of the compound, Raman microscopy was directly performed on the inclusions (Figure 4c). 
Acquired spectra showed the typical features assigned to lead antimonate doped with tin: in addition to the shifted $\mathrm{Pb}-\mathrm{O}$ lattice mode at $140 \mathrm{~cm}^{-1}$, a peak at about $450 \mathrm{~cm}^{-1}$, an increase in the band at about $330 \mathrm{~cm}^{-1}$, and a collapsed band at $510 \mathrm{~cm}^{-1}$ were observed, indicative of the partial replacement of the $\mathrm{Sb}^{+5}$ species by a larger $\mathrm{Sn}^{4+}$ cation $[28,29]$.

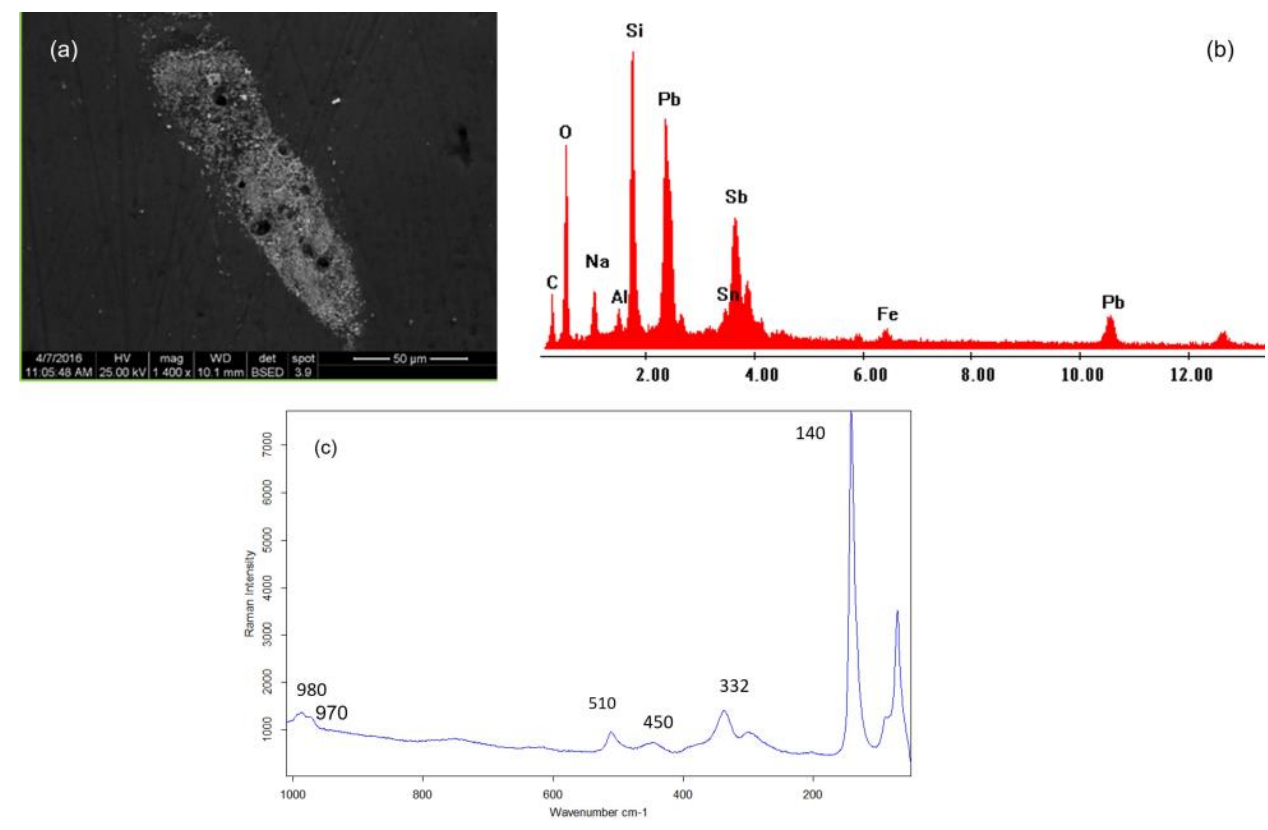

Figure 4. Example of lead-tin-antimony-based inclusions detected in a yellow mosaic glass tessera: (a) SEM-BSE image; (b) EDS spectrum; (c) Raman spectrum with bands at 140, 332, 450, and $510 \mathrm{~cm}^{-1}$ suggesting lead antimonate doped with tin [28,29].

In the above example, Raman microscopy can be considered as the most suitable analytical technique to gain data on the composition of the lead-tin-antimony-based compounds used to impart yellow colour and opacity to the glass. There are, however, cases in which the use of this analytical technique may not be informative. An example is represented by the opaque red coloured glass containing metallic copper inclusions. SEM-BSE images (Figure 5a) showed, at high magnification, nanometric rounded particles exclusively made of copper as EDS spot analysis demonstrate (Figure 5b). The nanometric size of these inclusions did not allow Raman microscopy analysis, where the magnifications of the most commonly accessible instruments hardly exceeds $1000 \times$. Diffractometric analysis (Figure 5c) can, in this case, be decisive, allowing one to distinguish the presence of metallic copper within the glass matrix. It should, of course, be emphasized that, unless instruments such as microdiffractometers and/or BSDE detectors interfaced with SEM are available, diffractometric analysis usually requires powdering the sample and, therefore, is destructive.

Once the investigation of colouring and opacifying phases was completed, we analyzed the bulk chemistry. To investigate the base glass, both in terms of compositional recipes and provenance of raw materials, a combination of Electron Probe Micro Analysis (EPMA) and Laser Ablation-Inductively Coupled Plasma-Mass Spectrometry (LA-ICPMS) analyses was proposed, together with specific data processing. Major and minor oxides, aimed at identifying both the fluxing agent and the "recipes" used in the glassmaking process, can be determined by EPMA, while LA-ICP-MS analysis needs to be carried out for measuring trace elements and, thus, drawing inferences on the provenance of the sands used as vitrifying agents. Several analytical techniques can be employed for the quantification of major, minor, and trace elements in archaeological and historical glasses, such as Wavelength Dispersive X-ray Fluorescence spectrometry (WDXRF), Inductively Coupled Plasma-Optical Emission Spectrometry (ICP-OES), Ion Beam Analysis (IBA), and Neutron Activation Analysis (NAA) [30]. However, when dealing with opaque coloured 
glasses the prime advantage of combining EPMA and LA-ICP-MS for quantification of major to trace elements is the possibility of performing both analyses on the same mounted and polished samples where the study of colourants and opacifiers had previously been carried out. When dealing with archaeological and historical glasses, it is important to identify "recipes" that can be linked to primary production furnaces and, therefore, provide information on geographical areas of production and provenance [3]. However, for deeply coloured glass, a further degree of difficulty is due to the "contamination" of the compositional data of the matrix with materials added as colouring and opacifying agents.
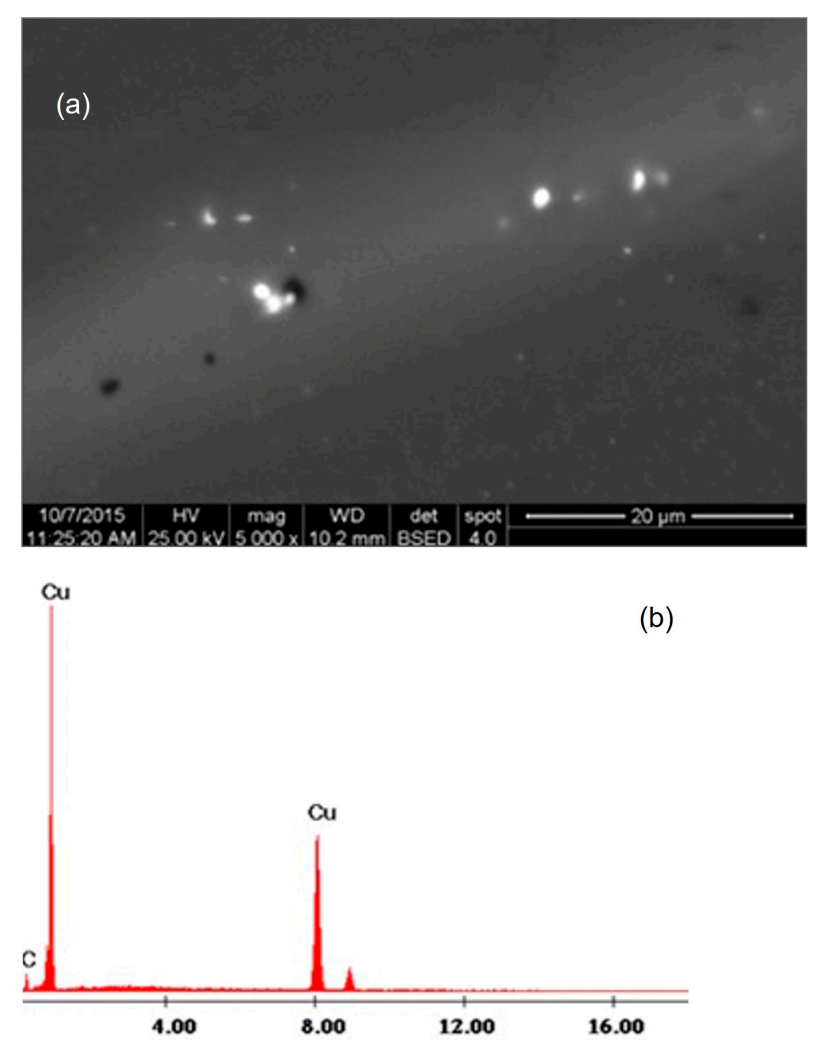

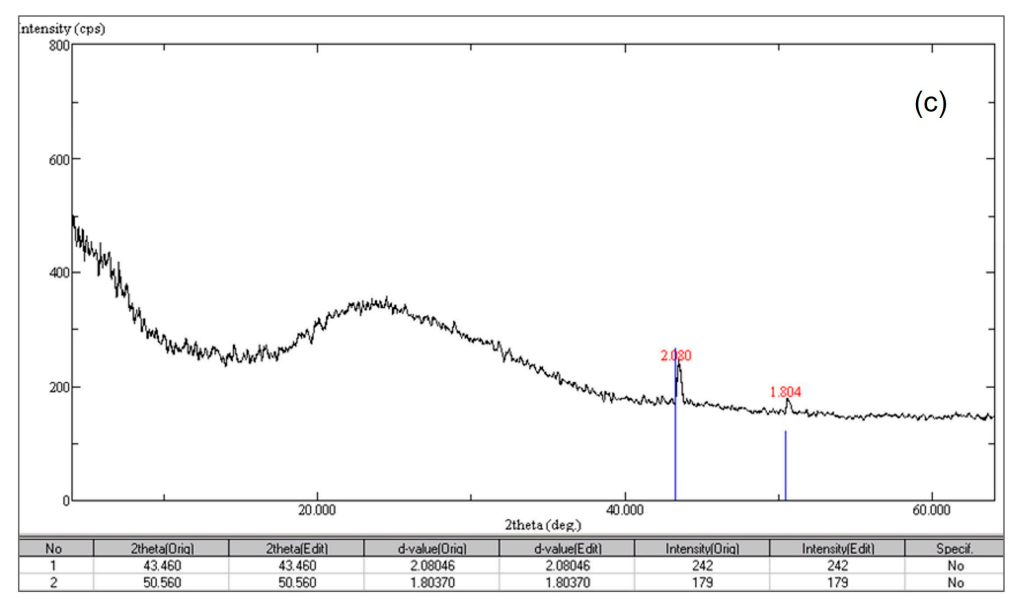

Figure 5. Example of nanometric copper-based inclusions detected in a red mosaic glass tessera: (a) SEM-BSE image; (b) EDS spectrum; (c) X-ray diffraction pattern showing the presence of metallic copper (Card No. standardreference4-0836).

To investigate the base glass, an integration between EPMA and LA-ICP-MS can work as a suitable compromise. Especially in the last few years, EPMA has been gradually being replaced by LA-ICP-MS for the determination of minor and major oxides, calculated by difference given a known oxide (generally $\mathrm{SiO}_{2}$ ). Recent research shows, indeed, that close correspondence is generally observed between the data achieved by EPMA and "new generation" LA-ICP-MS equipment when analysing colourless or naturally coloured glasses [31,32]. LA-ICP-MS can perform major to trace element analysis of almost all elements within a sample during a single run, due to specific quantification protocols such as Internal Standard Independent (ISI) and Sum Normalization (SN) methods [33]. Although the potentialities of this technique are significant, its application to the study of deeply coloured opaque glass still needs to be thoroughly explored. In particular, it should be noticed that the most commonly used quantification method (the Sum Normalization) assumes that glass is almost exclusively comprised of oxides in known oxidation states and that the sum of the concentration of all oxides should equal 100\% [33]. The first statement is quite difficult to verify when dealing with deeply coloured and opaque glasses such as tesserae, when the addition of several compounds is responsible for the colour shades and the opacity. Recent research also states that EPMA analysis of major and minor elements has several important advantages compared with other techniques, such as LA-ICP-MS as 
well as Secondary Ion Mass Spectrometry (SIMS): higher spatial resolution (from one to several $\mu \mathrm{m}$ ), a well-established matrix correction procedure, and a lower cost [34]. Given the current state of knowledge, further studies should, therefore, be conducted on the specific application of LA-ICP-MS for the quantification of not only trace, but also major and minor elements in opaque deeply coloured glass before a complete dismission of EPMA for quantitative analysis of major and minor elements can be proposed. When dealing with opaque deeply coloured glasses, EPMA data can, however, be affected by the addition of materials acting as colourants and opacifiers. To better compare the composition of the base glass with the categories reported in the literature for naturally coloured glass, EPMA data should, thus, be adequately processed in order to, if not completely neutralise, at least minimize this effect of contamination. Following the method proposed by Robert Brill [35], reduced composition can be obtained by subtracting the oxides of elements presumably due to additives from the total sum of all those measured and by normalising the remaining data. However, the main concern associated with Brill's method remains the following: when the recalculation is carried out, how can we be sure not to incur arbitrary subtractions? Previously performed in-depth characterisation of colouring and opacifying phases can provide considerable help and a treasurable guide in preventing any subjective subtractions, as we do know what materials are responsible for the hue and opacity of the samples under study and this information can guide us in the recalculation of data.

\section{Conclusions}

The meticulous connection and correlation between archaeological, typological, and compositional data is a key requisite when approaching the study of archaeological glass. An actual coparticipatory approach between archaeologists and archaeometrists stands, therefore, as a fundamental requisite for understanding objects beyond their chemistry and recognising them as witnesses of human material culture.

In the attempt to outline a best practice approach for the study of archaeological glass, the reasons for comprehending the contexts as the first step toward a tailored selection of materials have been discussed. Understanding if we are in a context with sound evidence of production/working activities will lead to selection criteria other than those that will guide the selection of objects from multilayered contexts (or where no univocal evidence of production has been ascertained).

A proper discernment of the archaeological context will, therefore, support a wellfitted selection of the type(s) of finds to be subjected to chronotypological study and archaeometric investigation. The analysis of compositional and technological features cannot, in fact, be separated from a careful and detailed chronotypological study, aimed at framing the object and its morphology in the related chronological and geographical context of diffusion.

Regarding archaeometric analyses, it can be stated that the setup of the analytical protocol may vary depending on the features of the objects to be analysed. When dealing with naturally coloured glass, the analytical approach can be based on the use of fewer analytical techniques, the primary objective being the determination of the chemical composition of the base glass and, if needed, the provenance of the materials used as vitrifying agents. Otherwise, deeply coloured (and often opaque) glass shows heterogeneity in terms of microstructure and compositional features, due to the addition of colouring and opacifying agents. The setup of a more articulated analytical approach is, thus, needed, based on the interrelation of different investigation techniques to achieve an in-depth characterisation of colouring and opacifying agents.

When dealing with opaque coloured glasses, the NCS System proved to be a suitable tool for a preliminary documentation of colours among assemblages to be studied: since NCS-coordinates describe the hue of opaque coloured glass, they can provide a first split of objects / fragment to be analysed into what can be defined as chromatic macrocategories (i.e., green, blue, red, and black), avoiding any subjective classification and denomination of the colours and, consequently, facilitating comparison among objects from different 
assemblages. By carrying out VIS-RS measurements, more in-depth information on optical properties related to different chromatic shades among coloured glasses belonging to the same chromatic macrocategory can, then, be achieved.

Since opaque coloured glasses show extremely heterogeneous microstructures, their microstructural and microtextural features should be investigated before the composition of the glassy matrix, to reduce the risk of interferences and misunderstandings in data evaluation. SEM-EDS is the starting point for the characterization of microstructural and microtextural features: BSE observations enable one to investigate and document the morphologies of the crystals precipitated into the glassy matrix, and EDS spot measurements provide preliminary data on their elemental composition. SEM-EDS is, thus, undoubtedly suitable to carry out high-resolution morphological inspection of the inclusions dispersed in a glassy matrix, as well as a qualitative and semiquantitative analysis of their elemental composition. However, if a more in-depth characterisation of these inclusions is needed to identify raw materials responsible for the colour and opacity of the samples under study, SEM-EDS inspection needs to be integrated with other techniques. As discussed in this paper, the choice of the most suitable technique/s can vary, depending upon the nature of the inclusions to be investigated. It has, for instance, been demonstrated that $\mu$ Raman can be extremely suitable for investigating the composition of lead-tin-antimony-based phases, but the same cannot be said for $\mathrm{Cu}$-based nanoparticles, where mineralogical analyses can be more informative.

In-depth examination of the microstructure needs to be followed by a determination of the bulk chemistry of the tesserae under study. Major and minor oxides, whose measurement is aimed at identifying both the fluxing agent and the "recipes" used in the glass-making process, have been analysed by EPMA. LA-ICP-MS analysis was carried out to determinine trace elements to draw inferences on the provenance of the sands used as vitrifying agents.

To identify, with as much precision as possible, the compositional categories of the base glass the tesserae belong to, EPMA data should always be recalculated (and normalised) to minimise any effect caused by any other intentionally added compound. A characterisation of the colouring and opacifying agents carried out prior to the analysis of the base glass will, therefore, also allow avoiding (or at least minimising) any subjective subtraction.

Last but not least, it should be remembered that, when dealing with archaeological materials, the study approach can be susceptible to implementations and variations evaluated on two fundamental criteria: the material features of the objects under study, which will never be identical to one another, as they are the product of ancient technologies and not of industrial processes; technological advancement and all that the progress of research will offer us which could be suitable to further deepen our knowledge of this material category.

Author Contributions: Conceptualisation, S.F. and T.C.; data curation, S.F.; methodology, S.F. and T.C.; resources, M.V.; supervision, M.V.; validation, M.V.; writing-original draft, S.F. and T.C. All authors have read and agreed to the published version of the manuscript.

Funding: This research received no external funding.

Institutional Review Board Statement: Not applicable.

Informed Consent Statement: Not applicable.

Data Availability Statement: The study does not report new data.

Acknowledgments: The authors sincerely thank the anonymous reviewers for their comments and valuable suggestions, which have contributed to increase the quality of the manuscript.

Conflicts of Interest: The authors declare no conflict of interest.

\section{References}

1. Artioli, G. Scientific Methods and Cultural Heritage: An Introduction to the Application of Materials Science to Archaeometry and Conservation Science; Oxford University Press: Oxford, UK, 2010.

2. Edwards, H.; Vandenabeele, P. Analytical Archaeometry: Selected Topics; Royal Society of Chemistry: London, UK, 2012. 
3. Rehren, T.; Freestone, I.C. Ancient glass: From kaleidoscope to crystal ball. J. Archaeol. Sci. 2015, 56, 233-241. [CrossRef]

4. Vandini, M.; Chinni, T.; Fiorentino, S.; Galusková, D.; Kaňková, H. Glass production in the Middle Ages from Italy to Central Europe: The contribution of archaeometry to the history of technology. Chem. Pap. 2018, 72, 2159-2169. [CrossRef]

5. Chinni, T.; Fiorentino, S.; Silvestri, A.; Vandini, M. Gambassini on the road. Underpinning evidence for a medieval widespread glass production in the north Adriatic area. J. Archaeol. Sci. Rep. 2021, 37, 103039. [CrossRef]

6. Fiorentino, S.; Vandini, M.; Chinni, T.; Caccia, M.; Martini, M.; Galli, A. Colourants and opacifiers of mosaic glass tesserae from Khirbet al-Mafjar (Jericho, Palestine): Addressing technological issues by a multi-analytical approach and evaluating the potentialities of thermoluminescence and optically stimulated luminescence da. Archaeol. Anthropol. Sci. 2019, 11, 337-359. [CrossRef]

7. Fiorentino, S. Fragile Connections, Persistent Methodology. A Tailor-Made Archaeometric Protocol to Investigate Technological and Cultural Issues in the Supply of Glass Tesserae under the Umayyad Caliphate. Ph.D. Thesis, University of Bologna, Bologna, Italy, 2019.

8. Vandini, M.; Fiorentino, S. From crystals to color: A compendium of multi-analytical data on mineralogical phases in opaque colored glass mosaic tesserae. Minerals 2020, 10, 609. [CrossRef]

9. Johnston-Feller, R. Color. Science in the Examination of Museum Objects: Nondestructive Procedures; The Getty Conservation Institute: Los Angeles, CA, USA, 2001.

10. Jarosewich, E. Smithsonian microbeam standards. J. Res. NIST 2002, 107, 681-685. [CrossRef]

11. Pouchou, J.; Pichoir, F. Determination of mass absorption coefficients for soft X-rays by use of the electron microprobe. In Proceedings of the 23rd Annual Conference of the Microbeam Analysis Society, Milwaukee, WI, USA, 8-12 August 1988; Newbury, D.E., Ed.; San Francisco Press: San Francisco, CA, USA, 1988; pp. 319-324.

12. Longerich, H.P.; Jackson, S.E.; Gunther, D. Inter-laboratory note. Laser ablation inductively coupled plasma mass spectrometric transient signal data acquisition and analyte concentration calculation. J. Anal. At. Spectrom. 1996, 11, 899-904. [CrossRef]

13. Kamber, B.S.; Greig, A.; Collerson, K.D. A new estimate for the composition of weathered young upper continental crust from alluvial sediments, Queensland, Australia. Geochim. Cosmochim. Acta 2005, 69, 1041-1058. [CrossRef]

14. Chinni, T. Produzione e Circolazione dei Manufatti In Vetro in Romagna nel Medioevo (V-XV sec.). Ph.D. Thesis, University of Bologna, Bologna, Italy, 2017.

15. Maltoni, S.; Chinni, T.; Cirelli, E.; Vandini, M.; Silvestri, A.; Molin, G. Archaeological and archaeometric study of the glass finds from the ancient harbour of Classe (Ravenna- Italy): New evidence. Herit. Sci. 2015, 3, 1-19. [CrossRef]

16. Maltoni, S.; Gallo, F.; Silvestri, A.; Vandini, M.; Chinni, T.; Marcante, A.; Molin, G.; Cirelli, E. Consumption, working and trade of Late Antique glass from north Adriatic Italy: An archaeometric perspective. In Things That Travelled. Mediterranean Glass in the First Millennium CE; Rosenow, D., Phelps, M., Meek, A., Freestone, I., Eds.; UCL Press: London, UK, 2018; pp. $191-214$.

17. Silvestri, A.; Molin, G.; Salviulo, G. The colourless glass of Iulia Felix. J. Archaeol. Sci. 2008, 35, 331-341. [CrossRef]

18. Silvestri, A. The coloured glass of Iulia Felix. J. Archaeol. Sci. 2008, 35, 1489-1501. [CrossRef]

19. Sedláčková, H.; Rohanová, D.; Lesák, B.; Šimončičová-Koóšová, P. Medieval glass from Bratislava (ca 1200-1450) in the context of contemporaneous glass production and trade contacts. Památky Archeol. CV 2014, 215-264.

20. Galli, A.; Poldi, G.; Martini, M.; Sibilia, E.; Montanari, C.; Panzeri, L. Study of blue colour in ancient mosaic tesserae by means of thermoluminescence and reflectance measurements. Appl. Phys. A Mater. Sci. Process. 2006, 83, 675-679. [CrossRef]

21. Galli, A.; Poldi, G.; Martini, M.; Sibilia, E. Thermoluminescence and visible reflectance spectroscopy applied to the study of blue-green mosaic silica-glass tesserae. Phys. Status Solidi Curr. Top. Solid State Phys. 2007, 4, 950-953. [CrossRef]

22. Maltoni, S.; Silvestri, A. A mosaic of colors: Investigating production technologies of roman glass tesserae from Northeastern Italy. Minerals 2018, 8, 255. [CrossRef]

23. Verità, M.; Maggetti, M.; Saguì, L.; Santopadre, P. Colors of Roman Glass: An Investigation of the Yellow Sectilia in the Gorga Collection. J. Glass Stud. 2013, 55, 21-34.

24. Schibille, N.; Neri, E.; Ebanista, C.; Ammar, M.R.; Bisconti, F. Something old, something new: The late antique mosaics from the catacomb of San Gennaro (Naples). J. Archaeol. Sci. Rep. 2018, 20, 411-422. [CrossRef]

25. Rosi, F.; Manuali, V.; Miliani, C.; Brunetti, B.G.; Sgamellotti, A.; Grygar, T.; Hradil, D. Raman scattering features of lead pyroantimonate compounds. Part I: XRD and Raman characterization of Pb2Sb2O7 doped with tin and zinc. J. Raman Spectrosc. 2009, 40. [CrossRef]

26. Molina, G.; Odin, G.P.; Pradell, T.; Shortland, A.J.; Tite, M.S. Production technology and replication of lead antimonate yellow glass from New Kingdom Egypt and the Roman Empire. J. Archaeol. Sci. 2014, 41, 171-184. [CrossRef]

27. Lahlil, S.; Cotte, M.; Biron, I.; Szlachetko, J.; Menguy, N.; Susini, J. Synthesizing lead antimonate in ancient and modern opaque glass. J. Anal. At. Spectrom. 2011, 26, 1040-1050. [CrossRef]

28. Rosi, F.; Manuali, V.; Grygar, T.; Bezdicka, P.; Brunetti, B.G.; Sgamellotti, A.; Burgio, L.; Seccaroni, C.; Miliani, C. Raman scattering features of lead pyroantimonate compounds: Implication for the non-invasive identification of yellow pigments on ancient ceramics. Part II. in situ characterisation of Renaissance plates by portable micro-Raman and XRF studies. J. Raman Spectrosc. 2011, 42. [CrossRef]

29. Paynter, S.; Kearns, T.; Cool, H.; Chenery, S. Roman coloured glass in the Western provinces: The glass cakes and tesserae from West Clacton in England. J. Archaeol. Sci. 2015, 62, 66-81. [CrossRef]

30. Janssens, K. Modern Methods for Analysing Archaeological and Historical Glass; John Wiley \& Sons Ltd.: Hoboken, NJ, USA, 2013. 
31. Gratuze, B. Glass characterization using Laser Ablation-Inductively Coupled Plasma-Mass Spectrometry methods. In Recent Advances in Laser Ablation ICP-MS for Archaeology; Dussubieux, L., Golitko, M., Gratuze, B., Eds.; Springer: Amsterdam, The Netherlands, 2016; pp. 179-196.

32. Ceglia, A.; Cosyns, P.; Schibille, N.; Meulebroeck, W. Unravelling provenance and recycling of late antique glass from Cyprus with trace elements. Archaeol. Anthropol. Sci. 2019, 11, 279-291. [CrossRef]

33. Cagno, S.; Hellemans, K.; Janssens, K. The Role of LA-ICP-MS in the Investigation of Archaeological Glass. In Recent Advances in Laser Ablation ICP-MS for Archaeology; Dussubieux, L., Golitko, M., Gratuze, B., Eds.; Springer: Berlin, Germany, 2016 ; pp. 163-178.

34. Batanova, V.; Sobolev, A.; Magnin, V. Trace element analysis by EPMA in geosciences: Detection limit, precision and accuracy. Mater. Sci. Eng. 2018, 304, 1-17. [CrossRef]

35. Brill, R.H. Chemical Analyses of Early Glass; Corning Museum of Glass: New York, NY, USA, 1999. 